\title{
Beam profile measurements based on modern vertex detectors and beam-gas interactions
}

\author{
Colin Barschel ${ }^{* \dagger}$ \\ CERN, Geneva, Switzerland \\ E-mail: colin.barschelecern.ch
}

\begin{abstract}
A novel, non-disruptive technique to measure transverse beam shapes was recently demonstrated by the LHCb experiment at the Large Hadron Collider (LHC). The technique is based on the detection of beam-gas interaction vertices with a tracking detector and was used in LHCb to obtain a $1.4 \%$ precision on the luminosity calibration. A new device, the Beam-Gas Vertex (BGV) system, is now under development to perform dedicated beam size measurements at the LHC at any beam energy and intensity. This technique could be applied to other particle accelerators. The BGV tracking detectors will be based on scintillating fibre modules read out by silicon photomultipliers. These modules are very similar in performance requirements and environmental constraints to the fibre tracker modules of the LHCb Upgrade and are therefore developed in close cooperation. The design studies, selected R\&D results and the expected performance of the BGV demonstrator system are presented.
\end{abstract}

Technology and Instrumentation in Particle Physics 2014,

2-6 June, 2014

Amsterdam, the Netherlands

* Speaker.

†n behalf of the LHCb collaboration. 


\section{Introduction}

A luminosity calibration is required at every new centre-of-mass energy $\sqrt{s}$ in order to evaluate the integrated luminosity of an acquired dataset. This calibration has to be performed at each experiment at the Large Hadron Collider (LHC). The instantaneous luminosity is a function of the colliding bunch pair properties and is given by

$$
L=v_{\mathrm{rev}} N_{1} N_{2} \Omega
$$

with $\Omega$ the beam overlap integral, $v_{\text {rev }}$ the collider revolution frequency and $N_{1}, N_{2}$ the number of particles in a bunch in beam 1 and 2, respectively. In the limit of ultra-relativistic particles (velocity close to the speed of light, $v \approx c$ ) and crossing at small angle, the beam overlap integral is given by

$$
\Omega=2 c \int \rho_{1}(x, y, z, t) \rho_{2}(x, y, z, t) \mathrm{d} x \mathrm{~d} y \mathrm{~d} z \mathrm{~d} t
$$

with $\rho_{1}(x, y, z, t)$ and $\rho_{2}(x, y, z, t)$ the particle density distributions of the two bunches.

The overlap integral is measured in dedicated experiments using two independent methods. The first method, the "van der Meer" scan method [1-5], measures the overlap integral by scanning the beams across each other in the vertical and horizontal plane. In this method the key principle is to express the overlap integral in terms of rates that are experimental observables as opposed to measure the bunch density functions directly. The second method, called "beam-gas imaging" (BGI) $[1,6]$, is based on beam-gas interactions vertices to measure the angles, positions and shapes of the individual beams without displacing them. The BGI method is, to date, a unique capability of the $\mathrm{LHCb}^{1}$ experiment.

The LHCb experiment is one of the four main experiments at the LHC at CERN [7,8] (European Organization for Nuclear Research). The experiment is dedicated to heavy-flavor physics, mainly to study CP violation and rare decays of charm and beauty hadrons; it is located in the former Delphi cavern at the LHC intersection point 8 . The relatively small mass of charm and beauty hadrons compared to the center-of-mass energy at the LHC, results in particle production concentrated in the forward or backward regions in a cone close to the beam pipe. To optimize the study of their decays, the detector is a single-arm spectrometer covering a forward angle of about $300 \mathrm{mrad}$. The detector geometry, which is optimized in the pseudorapidity range $1.8<\eta<4.9$, provides an almost ideal configuration to analyze beam-gas interactions.

An overview of the detector layout and its components is shown in Fig. 1. The VErtex LOcator (VELO) surrounds the interaction point (on the left in Fig. 1). The detector components with increasing distance to the collision point are the first ring-imaging Cherenkov (RICH) detector (RICH1), the tracker turicensis (TT), the magnet, the tracking stations (T1-T3), the second RICH detector (RICH2), the calorimeter system, which is composed of the scintillating pad detector (SPD), the pre-shower detector (PS) and the electromagnetic and hadronic calorimeters (ECAL and HCAL), and finally the muon stations (M1-M5).

\footnotetext{
${ }^{1}$ Large Hadron Collider beauty experiment.
} 


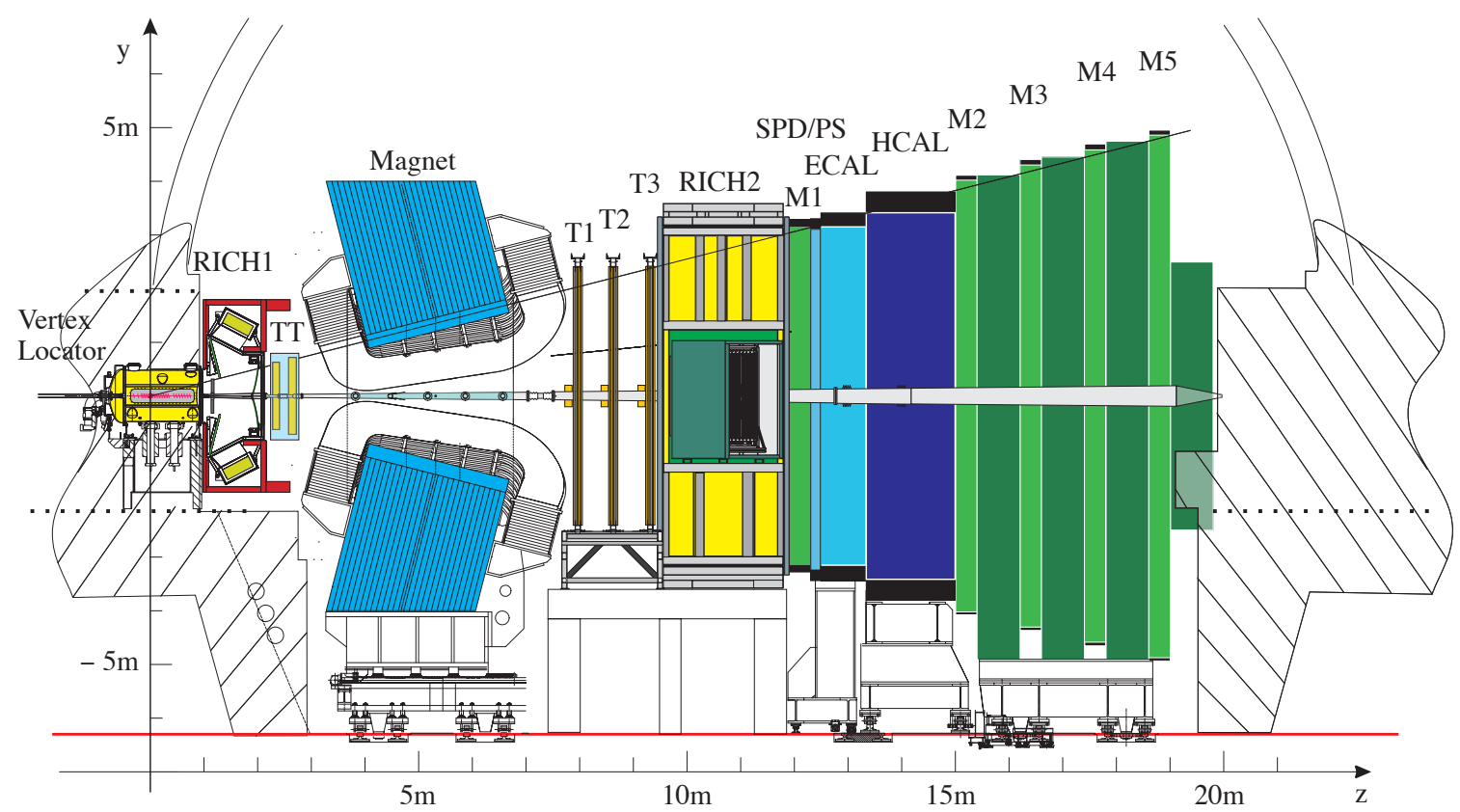

Figure 1: Vertical cross section through the LHCb detector. The different detector components are indicated in the figure (drawing from Ref. [7]).

\section{Beam-gas Imaging method}

The knowledge of the three-dimensional bunch shapes $\rho_{1,2}$ are required to evaluate the overlap integral defined in Eq. (1.2). The principle of the BGI method is to evaluate the overlap integral by measuring all required beam observables using the spatial distributions of beam-gas and beam-beam interactions.

During fills dedicated to luminosity measurements the normal vacuum pressure at the interaction point is $\sim 10^{-9}$ mbar, producing a beam-gas trigger rate of about $0.5 \mathrm{~Hz}$ per $10^{11}$ protons. Performing a BGI measurement with such low rates necessitates integration of a measurement over a period of up to 8 hours. Significant limitations in the precision are then caused by the low event rate, beam drift and by emittance growth over the integration time.

To increase the rate further and to take full advantage of the BGI capabilities, the use of a gas injection system was proposed [6], developed and commissioned in the LHCb experiment [9]. When activating the system, neon gas is injected in the VELO, thus raising the pressure from about $10^{-9}$ mbar to slightly above $10^{-7}$ mbar. The effect of gas injection on the pressure and beam-gas interaction rate is shown in Fig. 2. The pumps near the interaction point are switched off during the gas injection. With the trigger configuration chosen for $p p$ collisions at $\sqrt{s}=8 \mathrm{TeV}$, the beam-gas event rates per $10^{11}$ protons were $98 \mathrm{~Hz}$ for beam 1 and $82 \mathrm{~Hz}$ for beam 2 .

Vertex positions measured with the main LHCb silicon tracking detector (VELO) have an accuracy ranging from $10 \mu \mathrm{m}$ to $50 \mu \mathrm{m}$ depending on the vertex $z$ position along the beam axis, the number of reconstructed tracks, and whether the vertex originated from a beam-beam or beamgas interaction. The vertex resolution is therefore measured and parametrized independently for beam-beam interactions and beam-gas interactions for both beams as a function of track multiplicity, 

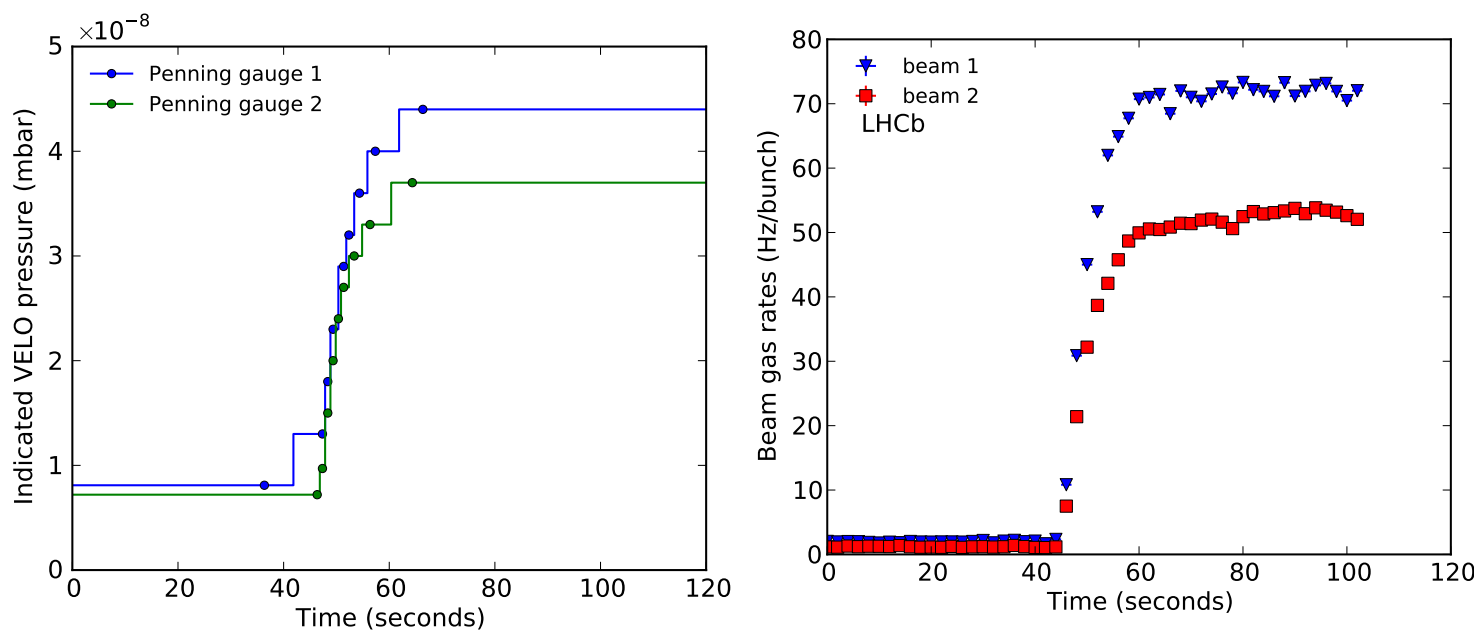

Figure 2: Beam-gas event rate increase with gas injection (fill 2520). Left: pressure increase in the VELO with gas injection. The pressure is measured with Penning gauges in the vacuum vessel within $50 \mathrm{~cm}$ of the interaction point. The indicated value when neon is injected is to be multiplied by 4.1 to account for the gauge sensitivity to neon. Right: selected beam-gas rate increase with gas injection. Here, the rate increases from about 2 to $72 \mathrm{~Hz} /$ bunch for beam 1 and 1.3 to $41 \mathrm{~Hz} /$ bunch for beam 2 compared to the vacuum condition with pumps switched off.

$z$ position and transverse axis. Resolution parametrization results for beam-gas interactions for beam 1 are shown in Fig. 3. Single beam profiles are measured by fitting the vertex distributions after deconvolving the VELO resolution, taking the beam directions and positions into account. An example of such a resolution unfolding is shown in Fig. 3. Final results are based on a twodimensional beam shape determination performed in a global fit including both beams and the luminous region.

Cross-section results for all dedicated luminosity calibration fills in 2012 at $\sqrt{s}=8 \mathrm{TeV}$ with $\beta^{*}=10 \mathrm{~m}$ beam optics and nominally head-on beams are shown in Fig. 4. The reference crosssection measured with the beam-gas imaging method has an uncertainty of $1.4 \%$ at $\sqrt{s}=8 \mathrm{TeV}$ [9]. This is, to date, the most precise luminosity calibration at the LHC and in any other bunched-beam proton collider. The precision reached in the beam-gas imaging measurements of the overlap integral relies on the gas injection system. The increased rate not only allowed the reduction of the integration time, but also permitted a two-dimensional shape analysis to be performed and provided a better accuracy in the resolution.

In addition to the beam shape measurement, the BGI method is also used to measure the charge fraction circulating outside of nominally filled bunches. This fraction is used to correct the bunch population product [10] and is an important information for all luminosity calibration fills.

\section{Beam Gas Vertex detector}

In light of the performances reached with the BGI method at LHCb, a new detector based on the beam-gas method is being developed to measure the beam size for the LHC. The Beam Gas Vertex (BGV) detector is designed as a non-disruptive device with the aim to measure individual 

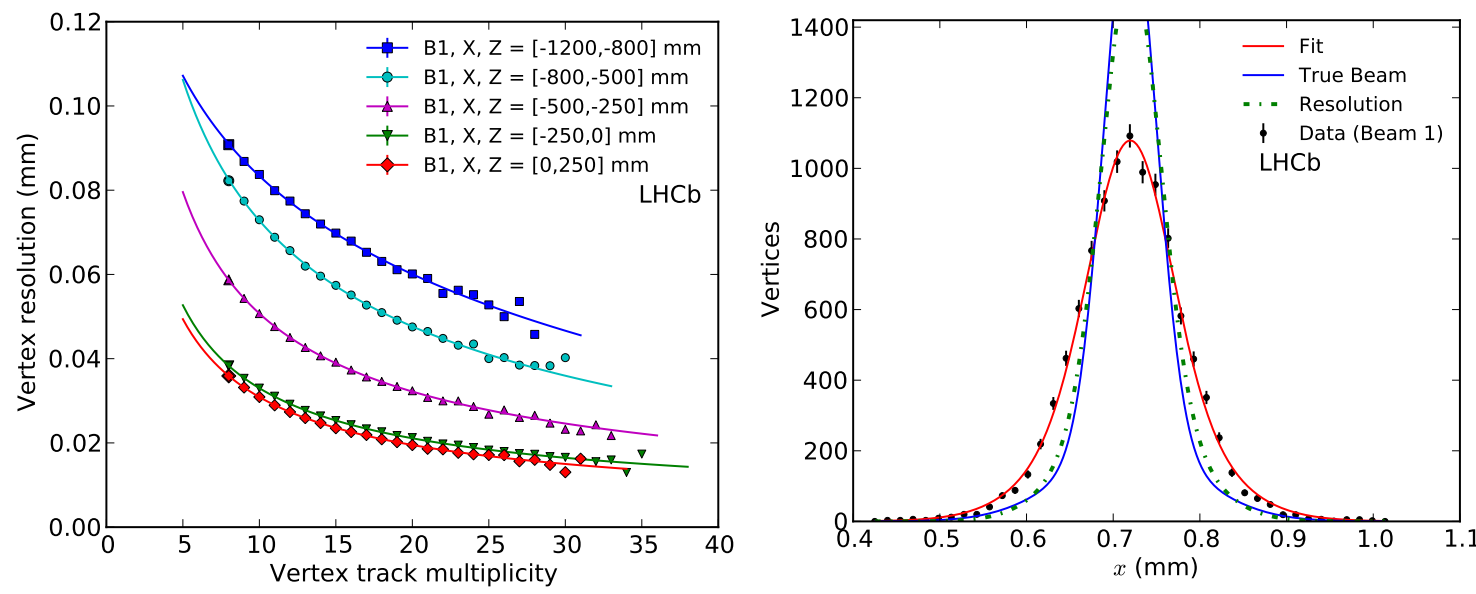

Figure 3: Left: Beam-gas vertex resolution in $x$ for five $z$ regions as a function of vertex track multiplicity for beam 1. Single markers indicate a resolution measurement per vertex track multiplicity. Continuous lines indicate the results of the fits using the parametrization directly in the same $z$ ranges. Results for the $y$ resolution are similar. Right: single beam shape measurement of beam 1 in $x$ for a fill with $\beta^{*}=3 \mathrm{~m}$. The true beam width is about $45 \mu \mathrm{m}$.
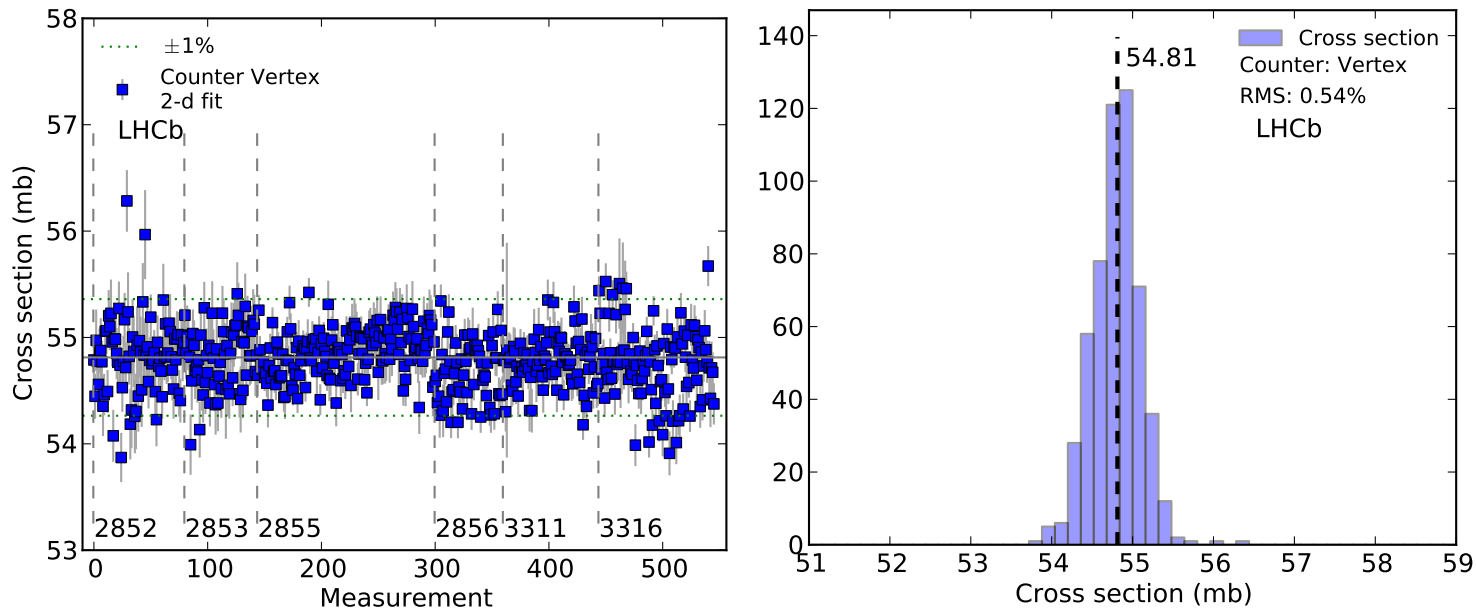

Figure 4: Cross-section results for head-on beams with $\beta^{*}=10 \mathrm{~m}$ beam optics at $\sqrt{s}=8$ TeV. Left: measurements are sorted by time and BCID (using the LHC naming). Each data point is a cross-section measurement from a colliding bunch pair using integrated data over about 20 minutes. The fills are indicated in the figure and are separated by a dashed vertical line. Two dotted horizontal lines indicate a $\pm 1 \%$ deviation from the central value. The error bars are the statistical uncertainty of the overlap integral. Right: results presented as histogram combining all data. 


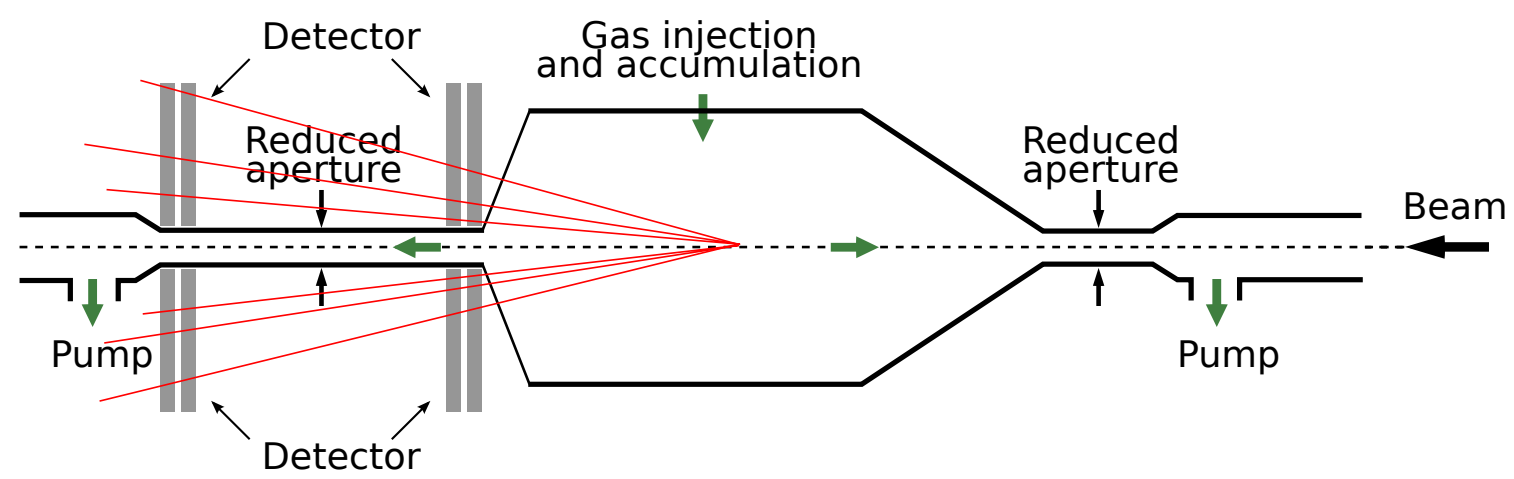

Figure 5: Layout of the BGV detector with the gas target at the center and the two detector planes on the left. An illustration of a beam-gas collision is shown in red.

bunch widths with a 5\% accuracy within 1 minute and to measure the average beam width with an accuracy of $2 \%$ within 1 minute [11]. The detector should be able to provide beam measurements throughout the LHC cycle including during the LHC energy ramp.

A collaboration between CERN, EPFL Lausanne, and RWTH Aachen has been formed to design and develop a prototype BGV detector that will be installed on one beam in the LHC. The general layout of the BGV system is shown in Fig. 5. The detector will use scintillating fibre (SciFi) modules, read out by silicon photomultipliers (SiPM). The modules are developed by the institutes EPFL, Lausanne and RWTH, Aachen. The detector technology is based on other projects from these institutes and, in particular, is used for the LHCb upgrade $[12,13]$.

In a similar way as for the LHCb experiment, the precision reached in the beam width measurement is essentially dictated by two main requirements, which are a precise vertex resolution and a sufficient beam-gas event rate. The number $N_{\text {good }}$ of events selected for a bunch width measurement can be estimated with

$$
N_{\text {good }}=f_{\text {good }} R_{\text {inel }} \Delta t \text {, }
$$

with $f_{\text {good }}$ the retention fraction of suitabe events, $R_{\text {inel }}$ the proton-gas inelastic rate and $\Delta t$ the integration time. The retention fraction depends on the detector acceptance and the rate depends on the gas pressure in the gas target and on the gas type. With an operational pressure of $6 \times 10^{-8} \mathrm{mbar}$ of neon and with a nominal LHC bunch intensity of $1.15 \times 10^{11}$ protons, the rate of inelastic beamgas interactions is expected to be about $100 \mathrm{~Hz}$. The retention fraction for neon is estimated to be $f_{\text {good }} \approx 0.02$.

The observed vertex distribution is a convolution of the physical beam with the detector resolution, that is, the knowledge of the beam-gas interaction position. Accordingly, the accuracy of the beam width measurement is determined by the vertex resolution. Considering the case where the shape of the beam as well as the vertex resolution can be described by a Gaussian function with a width of $\sigma_{\text {beam }}$ and $\sigma_{\mathrm{vtx}}$, respectively, the observed raw beam width can be described by $\sigma_{\text {raw }}^{2}=\sigma_{\text {beam }}^{2}+\sigma_{\mathrm{vtx}}^{2}$. Denoting the uncertainty on a measured value by $\delta$ and assuming a negligible uncertainty on the observed width $\left(\delta \sigma_{\text {raw }} \rightarrow 0\right)$, the relative uncertainty on the beam width measurement can be expressed by

$$
\frac{\delta \sigma_{\text {beam }}}{\sigma_{\text {beam }}}=\left(\frac{\delta \sigma_{\mathrm{vtx}}}{\sigma_{\text {beam }}}\right)^{2} \frac{\delta \sigma_{\mathrm{vtx}}}{\sigma_{\mathrm{vtx}}} .
$$


Both the knowledge of the resolution and a good resolution compared to the beam size are important to reach a precise measurement of the beam width.

The vertex resolution depends on the number of tracks $N_{\operatorname{Tr}}$ originating from a vertex and the impact parameter resolution $\sigma_{\mathrm{IP}}$ expected for a track. To first order the vertex resolution can be expressed as $\sigma_{\mathrm{vtx}}^{2}=\sigma_{\mathrm{IP}}^{2} / \sqrt{N_{\mathrm{Tr}}}$. The impact parameter depends on the multiple scattering $\sigma_{\mathrm{MS}}$ and the extrapolation error $\sigma_{\text {extrap }}$ and is given by $\sigma_{\mathrm{IP}}^{2}=\sigma_{\mathrm{MS}}^{2}+\sigma_{\text {extrap }}^{2}$. The extrapolation error depends on the hit resolution of the detector and the distance between the tracking stations. The performance of different detector geometries was studied to optimise the acceptance, the track impact parameter and vertex resolution.

Beam-gas interactions were simulated with the LHCb Computing Framework and the HIJING Monte Carlo generator [14]. A full Monte Carlo simulation is being developed using the LHCb software framework (Gaudi) [15]. For the most challenging conditions, a relative uncertainty of about $10 \%$ in the bunch width is expected at $6.5 \mathrm{TeV}$ with a normalized beam emittance of $3 \mu \mathrm{m}$ (see [16] and the references therein).

\section{Conclusion}

Several luminosity calibration measurements were performed with the beam-gas imaging method at LHCb using a gas injection system. An uncertainty of $1.4 \%$ at $\sqrt{s}=8 \mathrm{TeV}$ was reached at LHCb with the BGI method [9]. This measurement is, to date, the most precise luminosity calibration at the LHC and in any other bunched-beam proton collider. The gas injection system not only allowed the reduction of the integration time, but also the increased rate allowed to perform a two-dimensional shape analysis that was necessary to describe the beam shape. Furthermore, measurements provided by the BGI method with the gas injection system such as the unbunched charge fraction, the beam shape and its correlations have been used by the LHC experiments (ATLAS, ALICE, CMS, LHCb) and by the LHC machine.

The success of the beam-gas imaging method at LHCb lead to the development of the BGV demonstrator for the LHC. The BGV detector is being prepared for an installation in the LHC in 2014 and the commissioning is planned for 2015. The results of the BGV demonstrator will be used for the development of a more advanced BGV system for the High Luminosity LHC upgrade project. Furthermore, the experience gained in the operation of the SciFi detector will be used to identify possible constraints and to assess the aging performance of the long and large SciFi modules for the LHCb tracker.

\section{References}

[1] LHCb collaboration, R. Aaij et al., Absolute luminosity measurements with the LHCb detector at the LHC, JINST 7 (2012) P01010, arXiv:1110.2866.

[2] S. van der Meer, Calibration of the effective beam height in the ISR, Tech. Rep. CERN-ISR-PO-68-31. ISR-PO-68-31, CERN, Geneva, 1968. CERN-ISR-PO-68-31.

[3] C. Rubbia, Measurement of the luminosity of $p \bar{p}$ collider with a (generalized) Van der Meer Method,

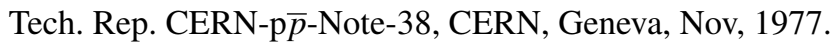


[4] V. Balagura, Notes on Van der Meer scan for absolute luminosity measurements, Nucl. Instrum. Meth. A654 (2011) 634.

[5] H. Burkhardt and P. Grafström, Absolute luminosity from machine parameters, Tech. Rep. CERN-LHC-PROJECT-Report-1019, CERN, 2007.

[6] M. Ferro-Luzzi, Proposal for an absolute luminosity determination in colliding beam experiments using vertex detection of beam-gas interactions, Nucl. Instrum. Meth. A553 (2005) 388.

[7] LHCb Collaboration, A. A. Alves et al., The LHCb Detector at the LHC, JINST 3 (2008), no. 08 S08005.

[8] LHCb Collaboration, R. Antunes-Nobrega et al., LHCb reoptimized detector design and performance: Technical Design Report, CERN, Geneva, 2003.

[9] C. Barschel, Precision luminosity measurements at LHCb with beam-gas imaging, PhD thesis, RWTH Aachen University, 2014, Presented 05 Mar 2014, CERN-THESIS-2013-301.

[10] A. Alici et al., Study of the LHC ghost charge and satellite bunches for luminosity calibration, CERN-ATS-Note-2012-029 PERF (2012), (BCNWG Note 4).

[11] P. Hopchev, A Beam Gas Vertex Detector for Beam Size Measurement in the LHC, Proceedings of 5th International Particle Accelerator Conference IPAC14 (2014).

[12] P. von Doetinchem et al., PEBS: Positron Electron Balloon Spectrometer, Nucl. Instrum. Meth. A581 (2007) 151, arXiv:astro-ph/0702567.

[13] LHCb Collaboration, R. Antunes-Nobrega et al., LHCb Tracker Upgrade Technical Design Report, no. CERN-LHCC-2014-001. LHCB-TDR-015 in Technical Design Report LHCb, CERN, Geneva, Feb, 2014.

[14] X.-N. Wang, HIJING Monte Carlo Model, http://ntc0.lbl.gov/ xnwang/hijing/.

[15] LHCb Collaboration, R. Antunes-Nobrega et al., LHCb computing: Technical Design Report, Technical Design Report LHCb, CERN, Geneva, 2005. Submitted on 11 May 2005.

[16] G. Bregliozzi et al., Beam Gas Vertex (BGV) Demonstrator of a Beam Profile Monitor in LHC, Tech. Rep. LHC-BGV-EC-0002-10-00, CERN, Geneva, Mar, 2014. 